\title{
Implicaciones de las proteínas de choque térmico (sHsp/HSPB) en el desarrollo de enfermedades degenerativas
}

\author{
Aura T. Chávez-Zobel*, Homero Sáenz-Suárez \\ ${ }^{1}$ Unidad De Biología Celular y Microscopía. Decanato de Ciencias de la Salud. \\ Universidad Centroccidental Lisandro Alvarado. Barquisimeto, Venezuela. \\ *chavezzobel@interlink.net.ve
}

Recibido: 30-04-2009; Aceptado: 27-08-2009

\begin{abstract}
Resumen
Las proteínas de choque térmico pertenecen al grupo de proteínas de estrés y son moléculas presentes en todas las células, se unen a los péptidos nacientes para dirigir su plegamiento, garantizando su estructura tridimensional y con ello su funcionamiento correcto. Dentro de éste grupo de moléculas se encuentran las proteínas de choque térmico pequeñas (sHsp/HSPB), también, capaces de unirse a péptidos y proteínas dañadas por diversos tipos de agresiones, facilitando su reparación o degradación. En células expuestas a situaciones adversas se produce un rápido aumento en las concentraciones de estas proteínas. Aunque inicialmente se asoció la expresión de las proteínas de estrés a aumentos bruscos de temperatura, hoy día es conocido que existen en condiciones fisiológicas normales y sus concentraciones se aumentan en respuesta a un amplio espectro de agresiones ambientales como: infecciones virales, inflamaciones, cuadros febriles, exposición a compuestos citotóxicos, acidificación del pH, anoxia o shock térmico. La producción de este tipo de moléculas constituye un mecanismo de defensa que permite a la célula adaptarse a condiciones anómalas y aumentar su capacidad de supervivencia. Se presenta en este trabajo, una breve reseña histórica de las proteínas de choque térmico pequeñas, su asociación con algunas patologías y se discute el estado actual del conocimiento de este tipo de moléculas y los posibles mecanismos implicados en las enfermedades conformacionales o proteinopatías, en donde existen alteraciones en la conformación nativa de las proteínas.
\end{abstract}

Palabras clave: Proteínas de choque térmico pequeñas (sHsp/HSPB), cuerpos de inclusión, enfermedades degenerativas, miopatías, mutaciones.

\begin{abstract}
The small heat shock proteins (sHsp/HSPB) and their implication in the development of degenerative diseases. Stress proteins are present in all the cells and participate in the synthesis of proteins binding their selves to the newly formed peptides to direct their folding, thus ensuring their three-dimensional structure and appropriate functioning. Besides, stress proteins are able to bind to damaged peptides and proteins due to diverse types of aggressions, enabling their repair or degradation. When cells are exposed to adverse situations, a rapid increase in concentration of stress proteins occurs. Stress protein expression had been associated to heat shocks only, but nowadays we know that stress proteins are induced as a response to a wide array of physiological and environmental aggressions such as: viral infections, inflammations, febrile responses, cell exposure to cytotoxic compounds, $\mathrm{pH}$ acidification, anoxia, and heat shock. The production of this type of molecules is a defense mechanism that allows the cell to adapt to anomalous situations and increase its survival capacity. In our study we present a brief historical account on stress proteins, their association with some pathologies, and discuss the current state of knowledge about this type of molecules and the possible mechanisms involved in protein conformational disorders or proteopathies.
\end{abstract}

Key words: Heat shock proteins (Hsp), body inclusion, degenerative diseases, myopathies, mutations. 


\begin{abstract}
Resumo
As proteínas pequenas de choque do calor (sHsp/HSPB) e sua implicação no desenvolvimento de doenças degenerative. As proteínas do estresse são moléculas presentes em todas as células e estão envolvidas no processo de síntese protéica, ligando-se aos peptídeos nascentes para dirigir o seu dobramento, o que garante sua estrutura tridimensional e, consequentemente, o seu bom funcionamento. Além disso, são capazes de se ligar a peptídeos e proteínas danificadas por diferentes tipos de agressão, facilitando a sua reparação ou degradação. Quando as células ficam expostas a situações adversas aumentam rapidamente as concentrações dessas proteínas. Embora no inicio associou-se a expressão das proteínas de estresse com uma mudança brusca de temperatura, atualmente é conhecido que elas são induzidas como resposta a um amplo espectro de agressões fisiológicas e ambientais, tais como: infecções virais, inflamações, febres, exposição das células a compostos citotóxicos, a acidificação do $\mathrm{pH}$, anoxia ou choque térmico. A produção de tais células é um mecanismo de defesa que permite à célula se adaptar a condições anormais e aumentar a sua capacidade de sobrevivência. Neste trabalho se apresenta um breve histórico das proteínas de estresse, a sua associação com certas doenças e se discute o estado atual do conhecimento sobre este tipo de moléculas e os possíveis mecanismos envolvidos nas doenças conformacionais ou proteinopatias.
\end{abstract}

Palavras chave: proteínas de choque térmico (Hsp), corpos de inclusão, doenças degenerativas, miopatias, mutações

\section{Introducción}

El estudio de las proteínas de estrés, entre las cuales se encuentran las proteínas de choque térmico, ha dado al mundo científico la oportunidad de descubrir múltiples senderos que aseguran la sobrevivencia celular tanto en condiciones fisiológicas como en condiciones de estrés. Aunque fue sólo en 1973, cuando Tissières y Mitchell identificaron las proteínas inducidas por el estrés (1), ya en 1962, Ritossa estudió indirectamente las proteínas de estrés al observar la activación transcripcional de los cromosomas de las glándulas salivales de Drosophila busckii, utilizando como estímulos el calor, el dinitrofenol y el salicilato de sodio, encontrando un aumento de proteínas de 70 y $26 \mathrm{kDa}$, las cuales fueron llamadas proteínas de choque térmico (HSP, Heat Shock Proteins) (1).

Evidencias experimentales, entre 1978 y 1979, demostraron que además de un choque térmico otros agentes de estrés inducían las HSP en bacterias, tetrahymena y células de pollo en cultivo. Desde entonces, se han acumulado múltiples pruebas científicas a favor del hecho de que la respuesta de estrés constituye un fenómeno altamente conservado, en prácticamente todos los tipos celulares. Esto último, ha contribuido a demostrar la importancia biológica de ese mecanismo de protección celular contra los efectos tóxicos inducidos por elementos estresantes (1-6).

Al inicio de la década de los 80 se demostró en diferentes sistemas celulares que la acumulación de las proteínas de estrés por medio de un choque térmico u otras formas de estrés coincide con un estado transitorio de la célula llamado termotolerancia (7-11). Las células se vuelven termotolerantes después de haber sufrido un ligero choque térmico; lo que significa que se hacen resistentes a un segundo choque térmico severo que sería mortal sin los efectos benéficos de las proteínas de estrés. Aunque para esta época, numerosos investigadores coincidían en que la respuesta de estrés tiene como finalidad la protección celular (1) y la importancia de las HSP en la sobrevivencia celular se iba haciendo cada vez más evidente, eran todavía desconocidos sus mecanismos de acción, que inhiben los efectos tóxicos inducidos por el estrés. Los esfuerzos investigativos en este sentido, durante los años 80, lograron, demostrar que Hsp60/HSPD1 y Hsp70/HSPA participan en la reparación y en la degradación de las proteínas dañadas durante un período de estrés, así como en la estabilización y el plegamiento de las proteínas nacientes. A todo lo largo de la década de los 80 y al comienzo de los años 90, se acumularon elementos de prueba que demostraron el rol de Hsp60/HSPD1 y Hsp70/HSPA como chaperonas moleculares dependientes del ATP (12-16).

En 1990, las proteínas de choque térmico de pequeña masa molecular (sHsps/HSPB, Small Heat Shock Proteins) eran ya sujeto de múltiples investigaciones. Los resultados obtenidos desde entonces, han demostrado que in vitro, esas proteínas actúan como chaperonas moleculares independientes del ATP (17). Esto implica que la actividad de chaperonas moleculares de esas proteínas difiere de la forma de actuación de Hsp60/HSPD1 y Hsp70/HSPA con el mismo propósito $(18,19)$.

En la actualidad se acepta que la actividad de chaperonas moleculares es la función principal de las proteínas de estrés, contribuyendo dicha actividad de manera importante a los mecanismos de acción termoprotectores de las HSP pequeñas. En el medio ambiente celular múltiples proteínas no pueden adquirir su conformación nativa sin la asistencia de las chaperonas moleculares (17). Las chaperonas moleculares se definen como "una familia de proteínas celulares las cuales median el plegamiento correcto de otros polipéptidos, y en algunos casos su ensamblaje en estructuras oligoméricas, pero no son componentes 
de las estructuras" (20). Por consiguiente, las chaperonas moleculares interactúan transitoriamente con otras proteínas a fin de impedir posibles interacciones no apropiadas conducentes a la agregación proteica. Igualmente, reconocen y se unen selectivamente a las proteínas que han perdido su conformación nativa formando complejos relativamente estables; estabilizan proteínas nacientes y las guardan en una conformación no plegada con la finalidad de permitir su translocación a través de las membranas, además, participan junto con otras familias de chaperonas moleculares en la recuperación de proteínas desnaturalizadas (21). Bajo los efectos del estrés, las proteínas que exponen superficies hidrofóbicas son reconocidas y protegidas por medio de la unión a chaperonas moleculares que impiden su agregación (22).

Existe un grupo importante de chaperonas moleculares implicadas en el plegamiento de las proteínas in vivo (21). Su masa molecular, su comportamiento celular y las funciones que realizan, definen las diferentes familias de HSP (22), entre las cuales se encuentran las Hsp100/HSPH, Hsp90/HSPC1, Hsp70/HSPA, Hsp60/HSPD1, Hsp40/ DNAJB1 y sHsp/HSPB. De otro lado, en función del consumo de energía para la realización de sus funciones, se consideran dos grupos, las dependientes de ATP: Hsp100/ HSPH, Hsp90/HSPC1, Hsp70/HSPA, Hsp60/HSPD1, Hsp40/DNAJB1 y las independientes de ATP: sHsp/HSPB $(16,17,24-29)$.

Los resultados obtenidos en numerosas investigaciones, durante los años 90, sugirieron que las proteínas de estrés se encuentran implicadas no sólo en procesos fisiológicamente normales, sino asociadas a procesos patológicos, lo cual abre una ventana de esperanza para la búsqueda de medidas terapéuticas basadas en la producción de este tipo de proteínas.

Proteínas de estrés y procesos patológicos:

\section{Proteínas de choque térmico (HSP)}

Ha sido demostrado reiteradamente que hay una sobreexpresión de las proteínas de estrés cuando se comparan enfermos y población sana. Se han estudiado los niveles de algunas proteínas de estrés y se han encontrado niveles elevados en pacientes con arterioesclerosis, arritmias, isquemia cardíaca, tejidos neoplásicos de mama, ovario, endometrio y sistema digestivo (30). De otro lado, un área de investigación biomédica muy interesante, resulta del estudio de las implicaciones de las HSP en enfermedades neurodegenerativas como Parkinson, Huntington y Alexander, en las cuales existen problemas de conformación proteica, por lo cual estas entidades se conocen como enfermedades conformacionales o proteinopatías $(31,32)$. Ese tipo de afecciones se caracteriza por la presencia de cuerpos de inclusión o agregados proteicos en las células, los cuales, de manera general, son el resultado de proteínas mal plegadas y en consecuencia de una sobrecarga en el sistema celular de control de la calidad proteica. En esos cuerpos de inclusión ha sido descrita la presencia de ubiquitina, de las sub-unidades del proteosoma y de chaperonas moleculares, elementos claves que participan en el proceso celular de control de calidad a nivel de las proteínas $(33,34)$.

La capacidad de las HSP de proteger contra la desnaturalización de las proteínas inducida por el estrés térmico y la asociación frecuente de las HSP con los agregados proteicos sugiere que las chaperonas moleculares pudieran jugar un rol importante en el desarrollo de las enfermedades neurodegenerativas. Es de hacer notar que aunque las enfermedades degenerativas en las que se encuentran implicadas las HSP, no constituyen un grupo de elevada incidencia, son enfermedades devastadoras que se manifiestan con la edad y son causa de elevados costos tanto económicos como afectivos para el paciente y su familia, que tiene que enfrentar tales problemas.

Mecanismos moleculares e implicaciones de las proteínas de estrés en enfermedades conformacionales:

\section{Actividad protectora de las HSP}

En C. elegans, se observó que la expresión de la proteína de fusión httQ82-GFP, la cual codifica la proteína huntingtina de 82 residuos de glutamina fusionada a la proteína GFP (Green Fluorescent Protein), induce la agregación de diversas proteínas con cadenas cortas de poliglutamina, lo que se traduce en un retardo en el desarrollo (35). Sin embargo, también se encontró que la coexpresión de Hsp104/HSPH1 y httQ82-GFP reduce la cantidad de agregados y permite el crecimiento normal del organismo, demostrando con esto el rol de Hsp104/HSPH1 en la protección celular.

Estudios posteriores (36), constataron que la proteína huntingtina con 72 residuos de glutamina (httQ72), se agrega cuando se expresa en células COS-1. Sin embargo, se demostró que el tratamiento con geldanamycina; un antibiótico que se une específicamente a Hsp90/HSPC1 y altera su función induciendo la degradación de proteínas que están mutadas en células tumorales (37); induce la síntesis de Hsp40/DNAJB1, Hsp70/HSPA1A y Hsp90/HSPC1 e impide la agregación de la proteína htt72Q. También fue determinado que la localización intracelular de htt72Q se mantenía asociada a estructuras granulares, en donde 
Hsp40/DNAJB1 y Hsp70/HSPA1A co-localizaban. Por el contrario, Hsp90/HSPC1 co-localizaba parcialmente con pequeños agregados. También hallaron que la proteína huntingtina con 51 residuos de glutamina (HD51Q), se distribuía uniformemente en el citoplasma cuando era coexpresada con Hsp40/DNAJB1 y Hsp70/HSPA1A. En función de esos resultados, es posible sugerir que Hsp40/ DNAJB1 y Hsp70/HSPA1A deben colaborar para impedir la formación de fibrillas amiloides características de las enfermedades donde hay agregación proteica (38).

\section{Proteínas de choque térmico pequeñas (sHsp/ HSPB)}

El estudio del efecto de las sHsp/HSPB sobre la toxicidad y la agregación, características que se manifiestan en pacientes que padecen enfermedades causadas por repeticiones de glutamina constituye un campo de investigación prometedor para el desarrollo de estrategias terapéuticas. La co-expresión de Hsp27/HSPB1 humana con las proteínas httEx1Q53, httEx1Q74 y httEx1Q103 (proteínas huntingtina que poseen 53, 74 y 103 residuos de glutamina, respectivamente) no bloquea la agregación, pero disminuye la toxicidad de esas proteínas, asociada a muerte celular, determinada por la presencia de núcleos fragmentados o condensados. La disminución de la toxicidad de la proteína httEx1Q53 depende de la fosforilación de Hsp27/ HSPB1. El mutante pseudo-fosforilado de la serina 15 (S15D) de Hsp27/HSPB1 (donde la serina ha sido cambiada por aspartato a fin de simular una proteína fosforilada) protege igual que la forma salvaje de la proteína, mientras que el mutante triplemente pseudo-fosforilado (S15D, S78D, S82D) no bloquea la muerte celular inducida por las proteínas httEx1Q74 y httEx1Q103. El efecto protector del doble mutante de fosforilación (S78D, S82D) se sitúa a medio camino entre el simple y triple mutante. Esta protección inducida por Hsp27/HSPB1 coincide con la inhibición de la activación de la caspasa-3, con la supresión de los radicales libres inducidos por la expresión de la proteína HttEx1Q74 y con el aumento del glutatión (39).

Mutaciones en las proteínas de choque térmico pequeñas (sHsp/HSPB):

\section{Alfa B-cristalina/HspB5}

El reciente descubrimiento de mutaciones en los genes de las sHsp/HSPB abre grandes posibilidades para el estudio de los genes que codifican estas chaperonas moleculares, aspecto de mucha importancia para develar como el producto de la mutación pudiera ser compensado y cuáles son las implicaciones de la ausencia o de las modificaciones de la función de esas chaperonas mutadas.
La mutación R116C en el gen de la $\alpha$ A-cristalina es responsable del desarrollo de cataratas de carácter autosómico dominante (40). El patrón cromatográfico reveló que la aA-cristalinaR116C migra como un oligómero de 2000 kDa comparativamente con 640 kDa de la proteína salvaje. También, se observó que en aA-cristalinaR116C la actividad de chaperona molecular se encuentra disminuida, lo que confirma que la estructura terciaria de aA-cristalinaR116C es alterada por la mutación (41). De otro lado, el oligómero de a-cristalina compuesto por $\mathrm{aA}$-cristalinaR $116 \mathrm{C}$ y aB-cristalina salvaje tiene una masa molecular aumentada y una sensibilidad térmica más elevada, debido a la exposición de las cadenas laterales de los residuos de triptófano. Además, a-cristalina posee una ligera reducción de su actividad de chaperona molecular y pérdida del $10 \%$ de su actividad protectora contra la agregación de la alcohol deshidrogenasa (42).

En 1998 (43), fue identificada una mutación en el gen de aB-cristalina salvaje/HSPB5 (CRYAB) como responsable de una miopatía ligada a los filamentos de desmina (Desminrelated myopathie o DRM), también llamada cristalinopatía. El gen CRYAB se encuentra en el cromosoma 11q21-23 y la transición heterozigótica del nucleótido 3787 conduce a la sustitución de la arginina en posición 120 (AGG) por una glicina (GGG). La enfermedad se manifiesta en la edad adulta, con debilitamiento de las partes proximales y distales de los músculos del cuello, tronco y paladar, cardiopatías y aparición de cataratas. Las biopsias de los pacientes con diagnóstico de DRM presentaban anomalías en zonas de las fibras musculares, donde se detectaba inmunoreactividad hacia aB-cristalina y desmina. La aBcristalinaR120G expresada en mioblastos C2.7 y en células BHK21, formó agregados citoplasmáticos y perinucleares en los que B-cristalina y desmina co-localizaban. Ultraestructuralmente se determinó que esos agregados formaban depósitos rodeados por filamentos de $10 \mathrm{~nm}$ de diámetro, característico de los filamentos intermedios (43).

Análisis realizados (44), sobre las anomalías presentes en las fibras cardíacas de ratones que sobre-expresaban aBcristalinaR120G y aB-cristalina salvaje, demostraron que la sobre-expresión de aB-cristalinaR120G, inducía la mortalidad de los ratones, alcanzándose el $100 \%$ a la edad adulta y que aunque la expresión de la forma salvaje no inducía ningún fenotipo en los cardiomiocitos, a un menor nivel de expresión de aB-cristalinaR120G los ratones presentaban un fenotipo similar a las cardiomiopatías ligadas a los filamentos de desmina. Estos ratones morían por problemas cardíacos entre 5 y 7 meses de edad, los corazones estaban dilatados y presentaban trombosis atrial y calcificaciones. Además, los ratones sufrieron congestión pulmonar y hepática, edema pleural, abdominal y ge- 
neral. La red de desmina de los cardiomiocitos se encontraba alterada, puesto que la desmina formaba agregados en lugar de estrías. También, se encontró que en los ratones de menos de tres meses de edad, la sobre-expresión del transgen de aB-cristalinaR120G inducía hipertrofia de los cardiomiocitos, caracterizada por aumento de su diámetro transversal. En los ratones de menos de seis meses de edad, esta hipertrofia se manifestó no solamente por aumento del diámetro sino también por alargamiento de las fibras, lo que provocó dilatación de los ventrículos y la falla cardiaca. Inicialmente, la hipertrofia fue compensada porque la función contráctil fue mantenida, pero se observó un déficit en el relajamiento de los ventrículos. Estos resultados demuestran que los ratones transgénicos que expresan aB-cristalinaR120G constituyen un buen modelo para el estudio de las cardiomiopatías ligadas a los filamentos de desmina.

Inicialmente, se reportó que la masa molecular de la proteína salvaje era de $650 \mathrm{kDa}$ y que aB-cristalinaR120G recombinante tenía una masa molecular de $1.4 \mathrm{MDa}$ (43), sin embargo, otros grupos de investigación han reportado masas moleculares de $720 \mathrm{kDa}(44)$ y de $823 \mathrm{kDa}$ (45), resultados que ponen de manifiesto la alteración de la estructura cuaternaria de la proteína. Adicionalmente, fue revelado mediante microscopía electrónica de transmisión (cryo-EM) que la estructura de aB-cristalinaR120G tiene un diámetro de $16 \mathrm{~nm}$, forma irregular y una cavidad central mal definida, mientras que por el contrario, B-cristalina salvaje tiene un diámetro de $13 \mathrm{~nm}$, forma esférica y una cavidad central mejor definida (41). Los resultados anteriores no dejan duda que la mutación R120G en aB-cristalina afecta la estructura de la proteína y por consiguiente su actividad de chaperona molecular.

Por otra parte, utilizando los filamentos intermedios GFAP (Glial Fibrillary Acidic Protein), para analizar su interacción con aB-cristalinaR120G, se encontró que aunque la proteína GFAP ensamblada in vitro es insoluble, en presencia de aB-cristalina salvaje o aB-cristalinaR120G, se tornó soluble hasta en 50 y $80 \%$, respectivamente. Lo cual sugiere una reducción de la capacidad de la proteína mutada para inhibir el ensamblaje de la proteína $\operatorname{GFAP}(46,47)$.

La unión de aB-cristalina salvaje a la proteína GFAP fue estudiada a 22,37 y $44^{\circ} \mathrm{C}$ y una pequeña proporción de aB-cristalina salvaje fue detectada en la fracción insoluble en todas las temperaturas analizadas. Por el contrario, aBcristalinaR $120 \mathrm{G}$ a 37 y $44^{\circ} \mathrm{C}$ sedimenta completamente con la proteína GFAP. Esta observación demostró que aBcristalinaR120G se une a los filamentos intermedios más fuertemente que la proteína salvaje. Un análisis por microscopía electrónica corroboró ese resultado puesto que toda la aB-cristalinaR120G sedimentó con los filamentos. Además, estudios de viscosimetría demostraron que aB-cristalina salvaje inhibió las interacciones no covalentes filamento-filamento y disminuyó la viscosidad del gel formado por el ensamblaje de los filamentos GFAP. La aBcristalinaR $120 \mathrm{G}$ no disminuyó esta viscosidad, demostrando así, la incapacidad de aB-cristalinaR120G para inhibir las interacciones filamento-filamento, a pesar que ella se une más fuertemente a la proteína GFAP. Esos resultados confirmaron la hipótesis planteada inicialmente por Vicart y colaboradores $(43,47)$, quienes señalaron que la mutación R120G de aB-cristalina afecta la capacidad de la proteína para organizar correctamente los filamentos de desmina, conduciendo así a la agregación de aB-cristalina y de desmina.

Con el propósito de analizar el comportamiento celular de aB-cristalinaR120G, empleando un vector con el gen de dicha proteína se transfectaron las líneas celulares NIH3T3, CCL39 y PTK2, observando en todos los casos la formación de cuerpos de inclusión perinucleares (48-50). Por medio de inmunomarcaje de la $\gamma$-tubulina (un marcador del centrosoma) se determinó que los cuerpos de inclusión se formaron en el centrosoma o próximos a él, en más del $90 \%$ de los casos. Estos agregados son estructuras amorfas similares a los agresomas (48-52). En los cuerpos de inclusión se observó la co-localización de aB-cristalinaR120G y ubiquitina. Es de hacer notar que en la mayoría de las enfermedades neurodegenerativas caracterizadas por la presencia de agregados proteicos, generalmente se observa la presencia de ubiquitina. Las células PTK2 son de origen epitelial y poseen dos redes de filamentos intermedios, una red formada por queratinas 8 y 18 y otra de vimentina. Estas células fueron analizadas por microscopía confocal después de ser transfectadas para expresar aBcristalinaR120G, observándose que tanto la queratina como la vimentina forman una especie de cesta alrededor de los agregados proteicos. Sin embargo, estos filamentos no se encontraron dentro de los cuerpos de inclusión, ni se observó perturbación de la red de filamentos intermedios. Esa estructura de los cuerpos de inclusión donde los filamentos intermedios rodean los agregados proteicos es lo que ha sido definido por Kopito y colaboradores como un "agresoma" (48,52). En tal sentido, un agresoma es considerado como un mecanismo empleado por las células para bloquear la precipitación de las proteínas, aislando del citoplasma aquellas que ya han precipitado (52).

En nuestro laboratorio (53), con el objeto de establecer el perfil de sedimentación de aB-cristalina salvaje y aB-cristalinaR120G en gradiente de glicerol fueron transfectadas células NIH3T3 con los plásmidos que codifican para la proteína $\mathrm{aB}$-cristalina salvaje y para aB- 
cristalinaR120G. Los resultados demostraron que aB-cristalina salvaje sedimentó con una masa molecular de 540 $\mathrm{kDa}$, mientras que aB-cristalinaR120G sedimentó como especies altamente heterogéneas de peso molecular de $2000 \mathrm{kDa}$ y más. Estas alteraciones estructurales se reflejaron en la pérdida de aproximadamente $70 \%$ de la actividad protectora de $\mathrm{aB}$-cristalina $\mathrm{R} 120 \mathrm{G}$ contra un choque térmico de $44^{\circ} \mathrm{C}$ durante 2.5 horas. Experimentos realizados con aB-cristalinaR $120 \mathrm{G}$ co-transfectada con aB-cristalina salvaje, Hsp27/HSPB1 y HspB8/HSP22, demostraron que la presencia de estas proteínas modula la formación de cuerpos de inclusión por aBcristalinaR120G. (48). Igualmente se determinó que cuando aB-cristalinaR120G fue co-expresada con aB-cristalina salvaje/HSPB5 y Hsp27/HSPB1 humana, el perfil de sedimentación mostró que aB-cristalinaR $120 \mathrm{G}$ recuperó su estructura cuaternaria nativa y la formación de cuerpos de inclusión perinucleares fue completamente bloqueada, observándose solamente precipitados pequeños y aislados en una cantidad reducida de células. Además, esas dos proteínas co-sedimentaron y co-precipitaron con aBcristalinaR120G, lo que sugiere que ellas forman heterooligómeros (53). Desde hace mucho tiempo se conoce que Hsp27 y aB-cristalina salvaje/HSPB5 tienen actividad de chaperonas in vitro (16). Sin embargo, el hecho que ellas interactúen con aB-cristalina salvaje/HSPB5 y $\mathrm{aB}$-cristalinaR $120 \mathrm{G}$ y que permanezcan asociadas con la estructura oligomérica final no permite determinar si la actividad de chaperonas moleculares está envuelta en el plegamiento, puesto que ha sido demostrado que las chaperonas moleculares no permanecen unidas a las proteínas que pasan por este sistema de control de calidad celular (17). En células PTK2 transfectadas para expresar solamente aB-cristalinaR120G, el análisis por Western blot mostró la proteína 50\% en la fracción soluble y $50 \%$ en la fracción insoluble. Puesto que ella es una proteína citoplasmática soluble (48), este hecho evidencia que se insolubiliza como consecuencia de la mutación aB-cristalinaR120G. Por definición una chaperona molecular no debe permanecer asociada con la estructura final, en tal sentido, fue analizado el efecto de HspB8/HSP22 (otra chaperona de pequeña masa molecular) sobre aB-cristalinaR120G. Los resultados mostraron que HspB8/HSP22 no formó hetero-oligómeros con aB-cristalinaR120G, co-inmunoprecipitó débilmente con aB-cristalina salvaje y Hsp27/HSPB1, mientras que lo hizo fuertemente con aB-cristalina R120G. Además, bloqueó la formación de los agregados, rescató la estructura oligomérica de aB-cristalinaR $120 \mathrm{G} \mathrm{a} 600 \mathrm{kDa}$ sin permanecer unida a la estructura final de aBcristalinaR120G. Estos hallazgos nos permiten sugerir que HspB8/HSP22 tiene la capacidad de reconocer proteínas mal plegadas (53).
En relación a la estructura y función de estas proteínas se hizo muy evidente la importancia de un residuo de arginina conservado en aA-cristalina (R116), en aB-cristalina (R120) y en Hsp27/HSPB1 de hamster (R148), el cual sustituido por Glicina (Hsp27R148G) provocó un fenotipo similar al observado en aB-cristalinaR120G, con la formación de agregados citoplasmáticos perinucleares y dímeros en lugar de los oligómeros de 12-24 sub-unidades de Hsp27/ HSPB1 salvaje, conservando su actividad de chaperona molecular tan alta como la proteína salvaje (54).

Posteriormente, han sido descritas otras dos mutaciones en el gen CRYAB, las cuales son responsables de Miopatía miofibrilar, caracterizada por degeneración progresiva de las miofibrillas que se inicia en los discos Z (55). En pacientes con esta patología a nivel de sus fibras musculares, ultraestructuralmente, se encontró acumulación de material filamentoso anormalmente degradado, pequeñas vacuolas autofágicas y acumulación aberrante de diferentes proteínas como desmina, aB-cristalina/HSPB5, distrofina, NCAM (Neural Cell Adhesion Molecule) y la kinasa CDC2 (55).

La secuenciación del gen CRYAB demostró una deleción de dos pares de bases en posición 464 (464delCT), que generó ocho codones sin sentido seguidos por un codón de parada. Esta mutación produjo una proteína truncada en su porción C terminal con 162 en lugar de 175 residuos y una masa molecular de $18.8 \mathrm{kDa}$. La segunda mutación debida a una transición $\mathrm{C} \rightarrow \mathrm{T}$ en posición $451(451 \mathrm{C} \rightarrow \mathrm{T})$, originó un codón de parada (Q151X), por lo cual el producto, en este caso, fue una proteína de 150 residuos y masa molecular de $17.5 \mathrm{kDa}$, mientras que el análisis del ADNc, de los dos pacientes estudiados, demostró la ausencia de splicing alternativo. Como consecuencia de estas dos mutaciones la aB-cristalina mutada pierde una parte significativa de su extensión C-terminal flexible, que es indispensable tanto para la solubilidad de la proteína, como para su función de chaperona molecular (55-57). Es interesante notar que un análisis de extractos celulares obtenidos a partir de músculo de los dos pacientes estudiados, demostró que aB-cristalina salvaje/HSPB5 y aB-cristalina mutada se encontraban presentes. Además, se pudo determinar que la expresión de aB-cristalina total se encontraba aumentada $35 \%$ en uno de los pacientes y $22 \%$ en el otro paciente, cuando se comparó con los individuos control, siendo la expresión de aB-cristalina mutada menor que la de aB-cristalina salvaje. En tal sentido, aB-cristalina mutada se comportó como un dominante negativo, puesto que a pesar de su reducida expresión, generó cambios patológicos en las fibras musculares. Por otra parte, se observó a nivel de los núcleos celulares de 7 a $8 \%$ de cambios morfológicos consistentes con alteraciones apoptóticas. 
Los autores discuten que la pequeña proporción de núcleos indicando muerte celular programada se explica considerando que aB-cristalina posee una función antiapoptótica al inhibir la Caspasa 3 (55).

Los resultados obtenidos por Simon y colaboradores (58, $59)$, ponen en evidencia que las proteínas mutantes aB-cristalinaQ151X y aB-cristalina464delCT forman agregados citoplasmáticos tal como los forma aBcristalinaR120G, que también fue analizada por estos investigadores.

Más recientemente ha sido descrita otra mutación en el gen CRYAB de aB-cristalina debido a la substitución de la base $\mathrm{G} \rightarrow \mathrm{A}$ que resulta en el reemplazo de ácido aspártico por asparagina en posición 140 (D140N). Esta mutación es autosómica dominante y su fenotipo es la formación de cataratas lamelares (60). Es de hacer notar que hasta la presente fecha no hay información adicional sobre la caracterización de la mutación.

\section{HspB8/HSP22}

La neuropatía motora conocida como Distal HMN (Distal Hereditary Motor Neuropathy) ha sido asociada con ocho loci y tres genes (61). El tipo II de la enfermedad es una alteración exclusiva de las neuronas motoras bajas, sin pérdida sensorial. En una familia checa y otra belga con diagnóstico de Distal HMN se identificó el cambio del nucleótido 423G0 C en el exón 2 de HspB8/HSP22, de forma que resulta en la sustitución del aminoácido lisina por asparagina en posición 141 (K141N). En otras dos familias, una búlgara y otra inglesa con el mismo diagnóstico se encontró la transición heterocigótica $421 \mathrm{~A} \rightarrow \mathrm{G}$ implicando el mismo residuo de lisina en una sustitución por glutamina (K141E). Estas mutaciones K141N y K141E, se encuentran localizadas en el dominio a-cristalino de HspB8/HSP22. Este residuo de lisina se encuentra conservado en diferentes especies y corresponde a la Arginina 120 de aB-cristalina que se encuentra mutada en DRM, lo que confirma la importancia de estos residuos conservados en esta familia de proteínas (61).

Un análisis de la capacidad de HspB8/HSP22 para bloquear la agregación proteica fue adelantado por Carra y colaboradores (62), quienes utilizaron como modelo la proteína huntingtina con 43 residuos de glutamina (htt43Q), puesto que se ha determinado que esta proteína con más de 37 residuos de glutamina forma agregados intracelulares insolubles (63). Para tal fin, células CCL39 fueron transfectadas con el plásmido pHDQ43-HA, solo o junto con el plásmido pMycHspB8. La actividad de HspB8/HSP22 fue comparada con la de otras chaperonas moleculares como Hsp27/
HSPB1, aB-cristalina/HSPB5, Hsp40/DNAJB1 y fue utilizada como control negativo CHIP (co-chaperona de Hsp70/ HSPA1B). Los resultados obtenidos demostraron que Hsp40/DNAJB1 tuvo un mayor efecto inhibitorio en cuanto a la agregación de htt43Q, observándose que 44 horas después de la transfección, Hsp40/DNAJB1 eliminó completamente htt43Q de la fracción SDS insoluble. La actividad de CHIP fue prácticamente nula al igual que las de Hsp27/HSPB1 y aB-cristalina/HSPB5. En contraste se observó que HspB8/HSP22 y Hsp40/DNAJB1 prácticamente eliminaron htt43Q de la fracción SDS insoluble. Resultados similares fueron encontrados utilizando la línea celular HEK-293, lo cual pone en evidencia que la actividad de HspB8/HSP22 es independiente de la línea celular utilizada (62).

En la misma línea celular CCL39 fue analizado el efecto de la sobre-expresión de HspB8/HSP22 sobre la formación de agregados por htt43Q. Las células transfectadas con el plásmido pHDQ43-HA (que codifica para la proteína huntingtina con 43 residuos de glutamina) forman agregados perinucleares en $90 \%$ de las células que expresan la proteína htt $43 \mathrm{Q}$. Sin embargo, también, se observó que algunas de esas células poseen material soluble, lo que se evidencia por el marcaje difuso de htt43Q. La sobre-expresión de htt43Q con HspB8/HSP22 en células CCL39 mostró un marcaje difuso de htt43Q con prácticamente total ausencia de agregados perinucleares. En aquellas células donde a pesar de la presencia de HspB8/HSP22 se observaron agregados formados por htt43Q, HspB8/HSP22 co-localizaba con htt43Q (62).

Utilizando la proteína htt $43 \mathrm{Q}$, fue analizado el efecto de las mutaciones K141N y K141E de HspB8/HSP22, sobre su actividad de chaperona molecular en células CCL39 y HEK-293, las cuales fueron transfectadas con un plásmido que codifica para htt43Q sola o con las dos proteínas mutadas de HspB8/HSP22. Los resultados obtenidos demostraron que las dos proteínas mutadas poseen reducida actividad de chaperonas moleculares. En efecto, htt $43 \mathrm{Q}$ co-transfectada con HspB8/HSP22 salvaje fue encontrada formando agregados en solo $10 \%$ de las células que expresan ambas proteínas. Mientras que en las células co-transfectadas con htt43Q y K141N o K141E de HspB8/HSP22, se encontró que aproximadamente $25 \%$ de las células que expresaban ambas proteínas contenían agregados (62).

\section{Hsp27/HSPB1}

La enfermedad axonal Charcot Marie Tooth (CMT), pertenece a las neuropatías hereditarias motrices y sensoriales y se caracteriza por degeneración de los nervios periféricos. Esta patología ha sido clasificada en CMT1 y CMT2 para 
diferenciar las formas desmielinizante y axonal respectivamente. La presentación CMT2 es una enfermedad genéticamente muy heterogénea y en la actualidad se han caracterizado ocho loci y cinco genes como responsables de la forma hereditaria de la misma. Evgrafov y colaboradores en el 2004 (64), estudiaron una familia rusa afectada con CMT2 y encontraron en el exón 2 de Hsp27/HSPB1 una transición heterozigota en posición $404(404 \mathrm{C} \rightarrow \mathrm{T})$ conducente a la mutación S135F. Además de la familia rusa, fueron analizados otros 301 individuos con diagnóstico de CMT y se realizó tamizaje de 115 individuos afectados con HMN, neuropatía periférica motora que se asemeja estrechamente a CMT2, exceptuando la ausencia de anormalidades sensoriales en HMN. La misma mutación $404 \mathrm{C} \rightarrow \mathrm{T}$ fue encontrada en otras cuatro familias rusas con CMT y en una familia inglesa con Distal HMN. Además, se encontraron otras cuatro mutaciones de Hsp27/ HSPB1 tanto en familias afectadas con CMT2 (S135F y R136W), como en familias diagnosticadas con Distal HMN (R127W, S135F, T151I y P182L). De las mutaciones encontradas en Hsp27/HSPB1, las mutaciones R127W, S135F, R136W y T151I se encuentran en el dominio alfacristalino de la proteína, mientras que la P182L se encuentra en la porción $\mathrm{C}$ terminal flexible de la proteína (55), región ésta que participa en el mantenimiento de la solubilidad de la proteína y en su función de chaperona molecular (55). Estas mutaciones descritas para Hsp27/ HSPB1 humana ocurren en residuos conservados en proteínas ortólogas de Hsp27 /HSPB1.

Tomando en consideración que las neuropatías periféricas hereditarias son causadas probablemente por pérdida axonal prematura debido a la degeneración neuronal, el equipo de Evgrafov (64), analizó el efecto de la mutación S135F en la sobrevivencia celular. A tal efecto, transfectaron células $\mathrm{N} 2 \mathrm{a}$, de neuroblastoma de ratón, con construcciones de ADN para Hsp27/HSPB1 salvaje unida a la proteína EGFP (Enhanced Green Fluorescent Protein) o con Hsp27S135F. Observaron que 48 horas después de la transfección, el número de células transfectadas con Hsp27 mutada era significativamente más bajo que el número de células transfectadas con Hsp27/HSPB1 salvaje, lo que no pudo ser atribuido a una expresión aumentada de la proteína mutada puesto que mediante análisis por Western blot se reveló que tanto Hsp27 salvaje como Hsp27 mutada tuvieron niveles similares de expresión. También pudieron observar la presencia de aproximadamente el doble de células N2a multinucleadas, cuando éstas expresaron Hsp27 mutada, en comparación con las células que expresaron Hsp27 salvaje. Además de lo antes mencionado, también se estudió el efecto de la mutación S135F de Hsp27 sobre el ensamblaje de los neurofilamentos (64). La proteína que forma la cadena ligera de los neurofila- mentos humanos (NF-L) puede auto-ensamblarse en filamentos homopoliméricos cuando se expresa en células SW13.cl.2Vim, una línea celular de carcinoma adrenal que no contiene una red endógena de filamentos intermedios. Cuando las células SW13.cl.2Vim son co-transfectadas con NF-L y Hsp27/HSPB1 salvaje, NF-L forma una red delgada de neurofilamentos y ocasionalmente se observan haces de filamentos gruesos. En el caso de las células SW13.cl.2Vim co-transfectadas con NF-L y Hsp27 mutada en $\mathrm{S} 135 \mathrm{~F}$, se observa que NF-L forma agregados y una menor cantidad de filamentos finos, lo cual sugiere que la mutación S135F afecta el ensamblaje de los neurofilamentos. El análisis de la mutación P182L permitió determinar que la misma es responsable de la formación de agregados citoplasmáticos insolubles dentro de los cuales se encuentran secuestradas las cadenas medianas de los neurofilamentos y dinactina p150, hecho que sugiere que el mecanismo implicado en la destrucción neuronal es la disrupción de componentes esenciales de la estructura axonal y de transporte (65).

En un estudio de 114 pacientes chinos diagnosticados con CMT autosómica dominante tipo II, se determinó, en cuatro familias, la presencia de la transición heterozigótica C379T en Hsp27 que conduce a la sustitución R127W, lo que sugiere que este cambio es responsable de la enfermedad CMT (66-68).

Más recientemente Houlden y colaboradores (69), reportaron para Hsp27/HSPB1 cinco mutaciones autosómicas dominantes y la primera mutación autosómica recesiva asociada con la enfermedad Distal HMN/CMT2. A excepción de $\mathrm{S} 135 \mathrm{~F}$, las otras mutaciones fueron reportadas por primera vez, siendo ocasionadas por cambios de nucleótidos. Así, C404T produce el cambio R140G; C116T ocasiona el cambio P39L; G250C determina el cambio G84R y C295A determina la mutación autosómica recesiva L99M. Es importante mencionar que en la región codificante de Hsp27/HSPB1 se encontró un cambio C379A responsable de la mutación silenciosa R127R, en un paciente con una sintomatología típica de Distal HMN, que constituye un caso esporádico. En este caso se podría especular que el simple cambio de un nucleótido es capaz de producir cambios en la conformación de la proteína que conllevan a la manifestación de los síntomas de la enfermedad, o bien que esta mutación no sea la responsable de la patología y el defecto molecular aun no haya sido determinado.

\section{Perspectivas}

Del análisis realizado anteriormente se desprende que las mutaciones hasta ahora descritas que afectan proteínas de 
la familia de las HSP están relacionadas especialmente con miopatías, enfermedades en las que se encuentran implicados los sistemas nervioso y muscular. En virtud de la importancia de dichas enfermedades varios grupos de investigación en el mundo, actualmente adelantan estudios en ésta área. En nuestro laboratorio, gran parte de los esfuerzos investigativos están dirigidos a la generación de información producto del estudio de mutaciones en las proteínas Hsp27/HSPB1, HspB8/HSP22, aA-cristalina/ HSPB4 y aB-cristalina/HSPB5, algunos independientes y otros en colaboración con otros grupos de investigación. Específicamente, estamos interesados en determinar las variaciones en la solubilidad de dichas proteínas, su ubicación intracelular y las interacciones que puedan encontrarse alteradas entre los distintos aminoácidos, para lo cual se adelantan estudios experimentales, acompañados de estudios de bioinformática, con propuestas de modelos computacionales tanto de las proteínas normales como de las mutadas, sus cambios estructurales y los posibles mecanismos de interacción presentes en procesos patológicos (datos no mostrados), lo que sin duda nos permitirá una mejor comprensión de los factores que contribuyen al desarrollo de las enfermedades en las cuales estas proteínas están implicadas. Todo el conocimiento construido con base en los resultados de los estudiosos de las proteínas de estrés, contribuirá de manera decidida, en el mediano plazo, a soportar los estudios conducentes a la búsqueda de medidas terapéuticas no convencionales para éste tipo de enfermedades que, aunque son muy poco frecuentes, resultan devastadoras para los pacientes que las padecen.

\section{Conflicto de intereses}

No existe conflicto de intereses entre los autores del presente trabajo.

\section{Referencias}

1. Linquist S. The heat-shock response. Annual Review of Biochemistry. 1986; 55, 1151-91.

2. Livak KJ, Freund R, Schweber M, Wensik PC, Meselson M. Sequence organization and transcription at two heat shock loci in Drosophila. Proceedings of the National Academy of Sciences of the United State of America. 1978; 75(11), 5613-17

3. Craig EA, McCarthy BJ, Wadsworth SC. Sequence organization of two recombinant plasmid containing genes for the major heat shock-induced protein of $D$. melanogaster. Cell. 1979; 16(3), 575-88.
4. Guttman SD, Gorovsky MA. Cilia regeneration in starved tethrahymena: an inducible system for studing gene expression and organelle biogenesis. Cell. 1979; 17(2), 307-17.

5. Lindquist S. Regulation of protein synthesis during heat shock. Nature. 1981; 293(5830), 311-14.

6. Miller MJ, Xuong NH, Geiduschek EP. Quantitative analysis of the heat shock response of Scharomyces cerevisiae. Jounal of Bacteriology. 1982; 151(1), 31127.

7. Landry J, Chretien P, Bernier D, Nicole LM, Tanguay RM. Thermotolerance and heat shock proteins induced by hyperthermia in rat liver cells. International Journal of Radiation Oncology Biology and Physics. 1982; 8(1), 59-62.

8. Landry J, Chretien P, de Muys JM, Rorais R. Induction of thermotolerance and heat shock protein synthesis in normal and respiration-deficient chick embryo fibroblast. Cancer Research. 1985; 45(5), 2240-47.

9. Chrétien P, Landry J. Enhanced constitutive expression of the $27-\mathrm{kDa}$ heat shock proteins in heat-resistant variants from Chinese hamster cells. Journal of Cellular Physiology. 1988; 137(1), 157-66.

10. Landry J, Chretien P, Lazlo A, Lambert H. Phosphorylation of Hsp27 during developpement and decay of thermotolerance in Chinese hamster cells. Journal Cell Physiology. 1991; 109(1), 7-15.

11. Samali A, Orrenius S. Heat shock proteins: regulators of stress response and apoptosis. Cell Stress \& Chaperones. 1998; 3(4), 228-36.

12. Longo F, Wang S, Narasimhan P, Zhang JS, Chen J, Massa SM, Sharp FR. cDNA cloning and expression of stress-inducible rat hsp70 in normal and injured rat brain. Journal of Neuroscience Research. 1993; 36(3), 325-35.

13. Hutchison KA, Dittmar KD, Czar MJ, Pratt WB. Proof that hsp70 is required for assembly of the glucocorticoid receptor into a heterocomplex with hsp90. Journal of Biological Chemistry. 1994; 269(7), 5043-49.

14. Hartl FU, Hlodan R, Langer T. Molecular chaperones in protein folding: the art of avoiding sticky situations. Trends in Biochemical Sciences. 1994; 19(1), 20-25. 
15. Bukau B, Horwich AL. The Hsp70 and Hsp60 chaperone machines. Cell. 1999; 92(3), 351-66.

16. Kampinga, HH, Hageman J, Vos MJ, Kubota H, Tanguay RM, Bruford EA, Cheetham ME, Chen B, Hightower LE. Guidelines for the nomenclature of the human heat shock proteins. Cell Stress Chaperones. 2009; 14(1); 105-11.

17. Ehrnsperger M, Gräber S, Gaestel M, Buchner J. Binding of non-native protein to Hsp25 during heat shock creates a reservoir of folding intermediates for reactivation. The EMBO Journal. 1997; 16(2), 221-29.

18. Horwitz J. á-crystallin can function as molecular chaperone Proceedings of the National Academy of Sciences of the United State of America. 1992; 89(21), 10449-53.

19. Horwitz J, Emmons T, Takemoto L. The ability of lens alpha crystalline to protect against heat-induced aggregation is age-dependent. Current Eye Research. 1992; 11(8), 817-22.

20. Ellis J. Proteins as molecular chaperones. Nature. 1987; 328(6129), 378-79.

21. Fink AL. Chaperone-mediated protein folding. Physiological Reviews. 1999; 79(2), 425-49.

22. Frydman J. Folding of newly translated proteins in vivo: the role of molecular chaperones. Annual Review of Biochemistry. 2001; 70, 603-47.

23. Slavotinek AM, Biesecker LG. Unfolding the role of chaperones and chaperonins in human disease. TRENDS in Genetics 2001; 17(9), 528-35.

24. Narberhaus F. ácrystallin-type heat shock proteins: socializing minichaperones in the contex of a multichaperone network. Microbiology and Molecular Biology Review. 2002; 66(1), 64-93.

25. De Jong WW, Leunissent JAM, Voorter CEM. Evolution of the á-crystallin/small heat-shock protein family. Molecular Biology and Evolution.. 1993; 10(1), 103-26.

26. De Jong WW, Capers GJ, Leunissen JAM. Genealogy of the á-crystallin-small heat shock protein superfamily. International Journal of Biological Macromolecules. 1998; 22(3-4), 151-62.

27. Krief S, Faivre JF, Robert P, Le Douarin B, BrumentLarignon N, Lefrère I, Bouzyk MM, Anderson KM,
Greller LD, Tobin FL, Souchet M, Bril A. Identificación and characterization of cvHsp. A novel human small stress protein selectively expressed in cardiovascular and insulin-sensitive tissues. The Journal of Biological Chemistry. 1999; 274(51), 36592-600.

28. Sugiyama Y, Suzuki A, Kishikawa M, Akutsu R, Hirose T, Waye MMY, Tsui SKW, Yoshida S, Ohno S. Muscle develops a specific form of small heat shock protein complex composed of MKBP/HspB2 and HspB3 during myogenic differentiation. The Journal of Biological Chemistry. 2000; 275(2), 1095-104.

29. Kappé G, Verschure P, Philipsen RLA, Staalduinen AA, Van de Boogart P, Boelens WC, de Jong WW. Characterization of the novel human small heat shock proteins: protein kinase-related HspB8/HSP22 and testis-specific HspB9. Biochimica and Biophysica Acta. 2001; 1520(1), 1-6.

30. Fontaine JM, Rest JS, Welsh MJ, Benndorf R. The sperm outer dense fiber protein is the $10^{\text {th }}$ member of the superfamily of mammalian small stress protein. Cell Stress and Chaperones. 2003; 8(1), 62-69.

31. Muchowski, PJ. Wacker JL. Modulation of neurodegeneration by molecular chaperones. Nature Reviews Neuroscience. 2005, 6, 11-12.

32. Arrigo AP, Simon S, Gilbert B, Kretz-Remy C, Nivon M, Czella A, Guillet D, Moulin M, Diaz-Latoud C, Vicart P. Hsp27 (HspB1) and áB-cristalina (HspB5) as therapeutic targets. FEBS Letters. 2007, 581, 366574.

33. Sherman MY, Goldberg AL. Cellular defenses against unfolded proteins: a cell biologis think about neurodegenerative diseases. Neuron. 2001; 29(1), 15-32.

34. Shinohara $\mathrm{H}$, Inaguyama $\mathrm{Y}$, Goto $\mathrm{S}$, Inagaki $\mathrm{T}$, Kato K. áB-crystallin and HspB8 are enhanced in the cerebral cortex of patients with Alzheimer's disease. Journal of the Neurological Sciences. 1993; 119(2), 203-38.

35. Satyal HSS, Schmidt E, Kitagawa K, Sondheimer N, Lindquist S, Kramer JM, Morimoto R. Polyglutamine aggregates alter protein folding homeostasis in Caenorhabditis elegans. Proceedings of the National Academy of Sciences of the United State of America. 2000; 97(11), 5750-55.

36. Sittler A, Lurz R, Lueder G, Priller J, Hayer-Hartl M. K, Hartl FU, Lehrach H, Wanker EE. Geldanamycin acti- 
vates a heat shock response and inhibits huntinting aggregation in a cell culture model of huntigton's disease. Human Molecular Genetics. 2001; 10(12), 1307-15.

37. Bedin M, Gaben AM, Saucier C, Mester J. Geldanamycin an inhibitor of the chaperone activity of Hsp90, induces MAPK independente cell cycle arrest. International Jounal of Cancer; 109(5), 643-52.

38. Wacker JL, Zareie MH, Fong H, Sarikaya M, Muchowski, P. Hsp70 and Hsp40 attenuate formation of spherical and annular polyglutamine oligomers by partitioning monomer. Nature Structural and Molecular Biology. 2006; 103(29), 11051-56.

39. Wyttenbach A, Sauvageot O, Carmichael J, DíazLatoud Ch, Arrigo AP, Rubinsztein D.C. Heat shock protein 27 prevents cellular polyglutamine toxicity and suppresses the increase of reactive oxygen species caused by huntingtin. Human Molecular Genetics. 2002; 11(9), 1137-51.

40. Litt M, Kramer P, LaMorticella D.M, Murphey W, Lovrien EW, Weleber RG. Autosomal dominant congenital cataract associated with a missense mutation in the human alpha crystalline gene CRYAA. Human Molecular Genetics. 1998; 7(3), 471-74.

41. Kumar LV, Ramakrishna TS, Mohan-Rao C. Structural and functional consequences of the mutation of a conservd arginine residue áA and áB crystallins. The Journal of Biological Chemistry. 1999; 274(34), 24137-41.

42. Bera S, Abraham EC. The áA-crystallin R116C mutant has a higher affinity for forming heteroaggregates with áB-crystallin. Biochemistry. 2002; 41(1), 297-305.

43. Vicart P, Caron A, Guicheney P, Li Z, Prévost M.C, Faure A, Chateau D, Chapon F, Tomé F, Dupret JM, Paulin D, Fardeau M. A missense mutation in the áBcrystallin chaperone gene causes a Desmin-Related Myopathy. Nature Genetics. 1998; 20(1), 92-95.

44. Wang K, Spector A. ATP causes small heat shock proteins to release denatured protein. European Journal of Biochemistry. 2001; 268, 6335-45.

45. Bova MP, Yaron O, Huang Q, Ding L, Haley D., Phoebe LS, Horwitz J. Mutation R120G in áB-crystallin, wich is linked to a Desmin Related Myopathy, results in an irregular structure and defective chaperone-like function. Proceedings of the National Academy of Sciences of the United State of America. 1999; 96(11), 6137-42.
46. Chowdary TK, Raman B, Ramakrishna T, and Rao M. Mammalian Hap22 is a heat-inducible-small-heatshocl protein with chaperone-like activity. Biochemical Journal. 2004; 381, 379-387.

47. Der Perng M, Cairns L, van den IJssel P, Prescott A, Hutcheson AM, Quinlan RA. Intermediate filament interactions can be altered by Hsp27 and áB-crystallin. Journal of Cell Science. 1999; 112, 2099-112.

48. Chávez-Zobel, AT, Loranger A, Marceau N, Thériault JR, Lambert H, Landry J. Distinct chaperone mechanism can delay the formation of aggresomes by the myopathy-causing R120G áB-crystallin mutant. Human Molecular Genetics. 2003; 12(13), 1609-20.

49. Johnston, JA, Ward, CI, Kopito RR. Aggresomes: a cellular response to misfolded proteins. Journal of Cell Biology. 1998; 143, 1239-54.

50. García-Mata R, Bebok Z, Sorscher EJ. Sztul E.S. Characterization and dynamics of aggresoma formation by a cytosolic GFP-chimera. Journal of Cell Biology. 1999; 146, 1239-54.

51. Wigley, WC, Fabunmi, RP, Lee, MG, Marino, CR, Maúllen, S, DeMartino, GN, Thomas, PJ. Dynamic association of proteosomal machinery with de centrosome. Journal of Cell Biology. 1999; 145, 481-90.

52. Kopito, RR, Sitia, R. Aggresomes and Russell bodies. Symptoms of cellular endigestion? EMBO Report. 2000; 1(3), 225-31..

53. Chávez-Zobel AT. Análisis de las mutaciones áBR120G de áB-cristalina y R148G de Hsp27 de hámster. Trabajo de Ascenso. Universidad Centroccidental Lisandro Alvarado. Barquisimeto, Venezuela. 2005; 1-172

54. Chávez-Zobel, AT, Lambert H, Thériault J R, Landry J. Structural instability caused by a mutation at a conserved arginine in the á-crystallin domain of Chinese hamster heat shock protein 27. Cell Stress and Chaperones. $2005 ; \mathbf{1 0}(2), 157-166$

55. Selcen D, Engel AG. Myofibrillar myopathy caused by novel dominant negative alpha B-crystallin mutations. Annals of Neurology. 2003; 54 (6): 804-10

56. Andley UP, Mathur S, Griest TA, Petrash JM. Cloning, expresión, and chaperone-like activity of human aA-crystallin. The Journal of Biological Chemistry. 1996; 271, 31973-80. 
57. Smulders RHP, Carver JA, Lindner RA, van Boekel MA, Bloemendal H, de Jong WW. Immobilization of the C-terminal extensión of bovine aA-crystallin reduces chaperone-like activity. The Journal of Biological Chememistry. 1996; 46, 29060-66.

58. Simon S, Michiel M, Skouri-Panet F, Lechaire JP, Vicart $\mathrm{P}$, Tardieu A. Residuo R120 is essential for the quaternary structure and functional integrity of human áBcrystallin. Biochemistry. 2007a; 46(33), 9605-14.

59. Simon S, Fontaine JM, Martin JL, Sun X, Hoppe AD, Welsh MJ, Benndorf R, Vicart P. Myopathy-associated áB-crystallin mutants abnormal phosphorylation, intracellular location, and interactions with other small heat shock proteins. The Journal of Biological Chemistry. 2007b; 282(47), 34276-87.

60. Liu Y, Zhang X, Luo L, Wu M, Zeng R, Cheng G, Hu B, Liu B, Liang JJ, Shang F. A novel áB-crystallin mutation associated with autosomal dominant congenital lamellar cataract. Investigative Ophthalmology and Visual Science. 2006; 47, 1069-75.

61. Irobi J, Vam Impe K, Seeman P, Jordanota A, Dierick I, Verpoorten N, Michalik A, De Vriendt E, Jacobs A, Van Germen V, Vennekens K, Mazanec R, Tournev I, Milton-Jones D, Talbot K, Kremensky I, Van Den Bosch L, Robberecht W, Vandekerckhove J, Van Broeckhoven C, Gettemans J, De Jonghe P, Timmerman V. Hot-spot residue in small heat-shock protein 22 causes distal motor neuropathy. Nature Genetics. 2004; 36(6), 597-601.

62. Carra S, Sivilotti M, Chávez Zobel A, Lambert H, Landry J. HspB8, a small heat shock mutated in human neuromuscular disorders, has in vivo chaperone activity in cultured cells. Human Molecular Genetics. 2005; 14(12), 1659-69.

63. Ross CA, Poirier MA, Wanker EE, Amzel M. Polyglutamine fibrillogenesis: the patway unfolds. Proceedings of the National Academy of Sciences of the United State of America. 2003; 100, 1-3
64. Evgrafov OV, Mersiyanova I, Irobi J, Van Den Bosch L, Dierich I, Leung CL, Schagina O, Verpoorten N, Van Impe K, Fedotov V, Dadali E, Auer-Grumbach M, Windpassinger C, Wagner K, Mitrovic Z, Hilton-Jones D, Talbot K, Martin JJ, Vasserman N, Tverskaya S, Polyakov A, Liem RK, Getteman J, Robberechit W, De Jong P, Timmerman V. Mutant small heat-shock protein 27 causes axonal Charcot-Marie-Tooth disease and distal hereditary motor neuropathy. Nature Genetics. 2004; 36(6), 602-6.

65. Ackerley S., James P.A, Kalli A, French S, Davies KE, and Talbot K. A mutation in the small heat-shock protein HspB 1 leading to distal hereditary motor neuronopathy disrupts neurofilament assembly and the axonal transport of specific cellular cargoes. $\mathrm{Hu}$ man Molecular Genetics. 2006; 15(2), 347-54.

66. Fontaine JM, Sun X, Hoppe AD, Simon S, Vicart P, Welsh M, Benndorf R. Abnormal small heaat shock protein interactions involving neuropathy-associated Hsp22 (HspB8) mutants. The FASEB Journal. 2006; 20, E1579-E88,

67. Tang BS, Zhao GH, Luo W, Xia K, Cai F, Pan Q, Zhang RX, Zhang FF, Liu XM, Chen B, Zhang C, Shen L, Jiang H, Long ZG, Dai HP. Small heat-shock protein 22 mutated in autosomal dominant Charcot-MarieTooth disease type 2L. Human Genetics. 2005a; 116(3), 222-24.

68. Tang B, Liu X, Zhao G, Luo W, Xia K, Pan Q, Cai F, Hu Z, Zhang C, Chen B, Zhang F, Shen L, Zhang R, Jiang H. Mutations analysis of the small heat shock protein 27 gene in Chinese patients with CharcotMarie-Tooth disease. Archives of Neurology. 2005b; 62, 1201-07.

69. Houlden H, Laura M, Wavrant-De Vrièze F, Blake J, Wood N, Reilly M.M. Mutations in the Hsp27 (HspB1) gene cause dominant, recessive, and sporadic distal HMN/CMT type 2. Neurology. 2008; 71(21), 1656-57. 